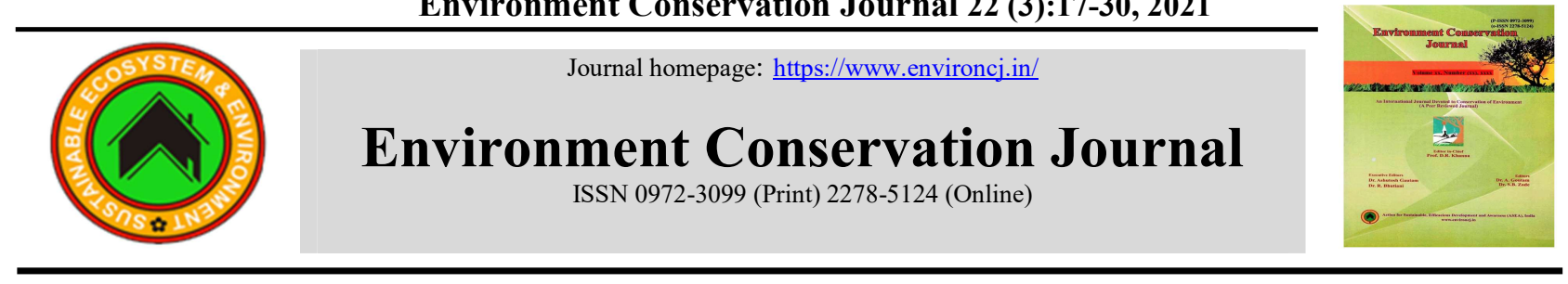

\title{
Effect of differential nitrogen management practices on growth, yield and water use efficiency of rice under varying moisture regimes
}

\author{
Barnali N \\ Department of Agronomy, College of Agriculture, CAU, Imphal, Manipur, India \\ L. Nabachandra Singh \\ Department of Agronomy, College of Agriculture, CAU, Imphal, Manipur, India \\ Ingudam Bhupenchandra 鴈 \\ ICAR-KVK Tamenglong, ICAR Research Complex for NEH Region, Manipur Centre, Lamphelpat, Manipur, India \\ S.K. Chongtham \\ Multi Technology Testing Centre and Vocational Training Centre, CAEPHT, CAU, Ranipool, Gangtok, Sikkim, India \\ L.C. Olivia \\ Pandit Deen Dayal Institute of Agricultural Sciences, Utlou, Bishnupur District, Manipur, India \\ E. Lamalakshmi Devi \\ ICAR-RC for NEH Region, Sikkim Centre, Tadong, Gangtok, India \\ Soibam Sinyorita \\ Department of Agronomy, College of Agriculture, CAU, Imphal, Manipur, India \\ Soibam Helena Devi \\ Department of Crop Physiology, Assam Agricultural University, Jorhat, Assam \\ Reaya Chanu \\ Multi Technology Testing Centre and Vocational Training Centre, CAEPHT, CAU, Ranipool, Gangtok, Sikkim, India \\ Merinda Wangkheimayaum \\ Multi Technology Testing Centre and Vocational Training Centre, CAEPHT, CAU, Ranipool, Gangtok, Sikkim, India
}

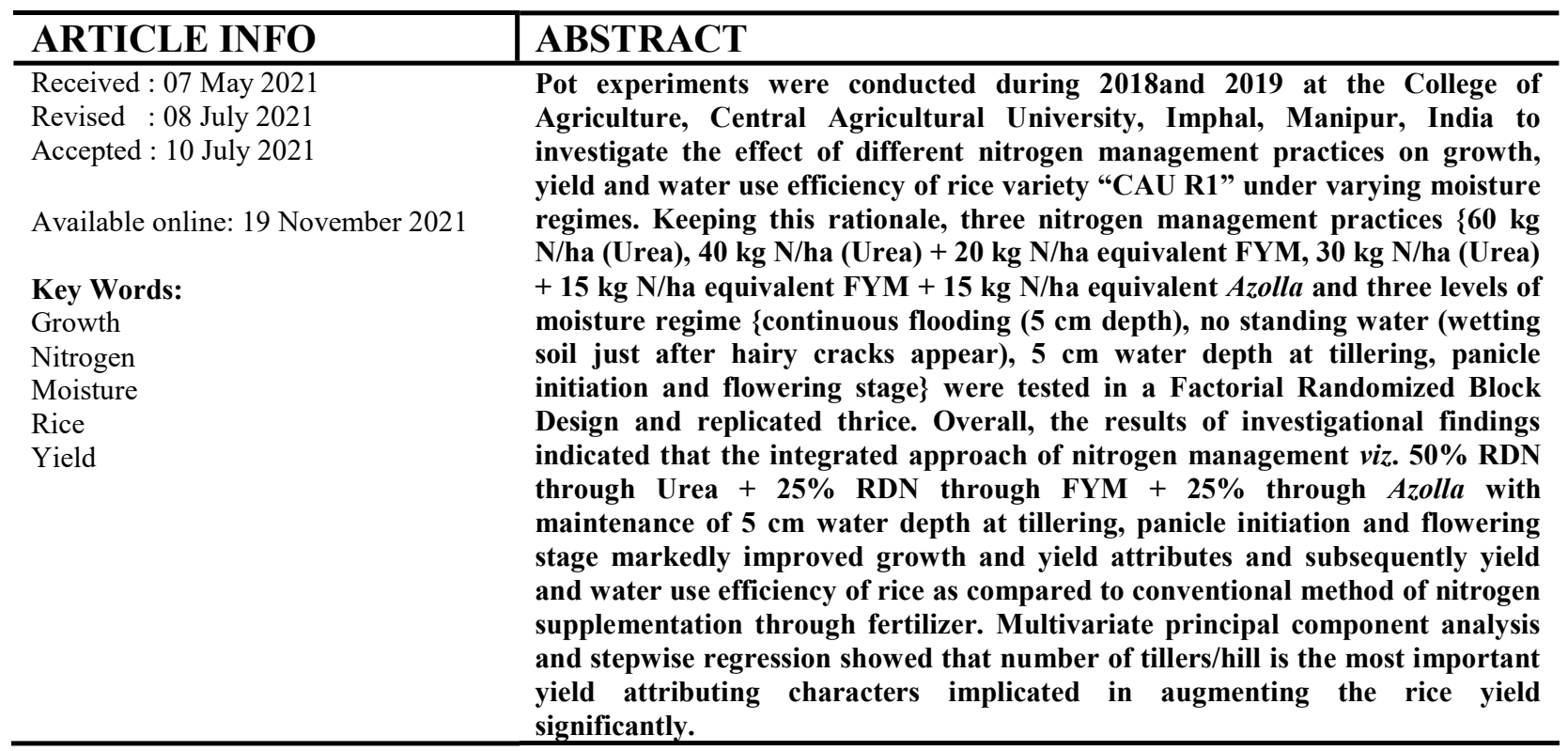

\section{Introduction}

Rice (Oryza sativa L.) is one of the most important cultivation is under a serious crisis globally due to starchy grain crops of the world which feeds about several constraints including stagnating yields, a half of the Earth's population. However, its dwindling area, rising input costs, shortage of water

Corresponding author E-mail: ibhupenj@gmail.com

Doi: https://doi.org/10.36953/ECJ.2021.22303

This work is licensed under Attribution-Non Commercial 4.0 International (CC BY-NC 4.0)

(C) ASEA 
and labour (Thakur et al., 2016). This further adds a tremendous onus on the shoulders of the rice growers to produce adequate tonnes of rice to feed the burgeoning population of the world. In this context, exploiting the production potential of rice through efficient agronomic management is one of the alternatives to safeguard and sustain food security. Nitrogen $(\mathrm{N})$ and water management are two production factors which play significant role in enhancing the rice production (Jat et al., 2020). $\mathrm{N}$ is one of the major essential macro-nutrients of plants, which insufficient availability in soil limits the crop yield. Application of inorganic $\mathrm{N}$ fertilizers has contributed substantially to the spectacular increase in agricultural production. However, low fertilizer N-use efficiency, ranging from 18 to $40 \%$, has been registered in rice soils due to rapid loss of applied inorganic $\mathrm{N}$ from the soil through various processes like ammonia volatilization and denitrification, which further contributes in environmental pollution. Also, continuous and disproportionate application of chemical fertilizers has been attributed to soil contamination and nutrient stress (Bisht and Chauhan, 2020). So, there arises an obligation to formulate ways to endure and enhance the overall productivity of rice-based cropping systems for better livelihood. Sustainable practices like integrated use of both organic and inorganic fertilizers have been often regarded as a viable alternative to address this problem (Yadav and Meena, 2014). The conjunctive use of organic manures and chemical fertilizers ensures the supply of essential nutrients in adequate amount and proportion, improves nutrient uptake by altering the soil physical behaviour and enhances the useefficiency of native and applied nutrients (Sharma et al., 2019), and restores the soil health (Kumrawat et al., 2019). Therefore, the integrated use of organic manure and inorganic fertilizer will restore the soil health and improve the performance in crops like rice by sustaining higher yield. Probably, there is no universally acceptable formula of integrated nutrient management practice. Thus, more efforts are needed to identify the improved nutrient management strategy for a particular target environment. Multivariate statistical tools like principal component analysis can identify significant yield attributing characters influencing the rice yield and, these results can now be effectively resorted by breeders to develop high yielding rice varieties and novel breeding procedures for rice improvement in the future (Maji, 2012).

Rice cultivation consumes more than $50 \%$ of the total fresh water utilised under irrigated agriculture in the world (Jat et al., 2016). It is estimated that about 3000-5000 litres of water are utilized to yield one $\mathrm{kg}$ of rice (Jat et al., 2020). However, water is becoming increasingly scarce because of population explosion, rapid urban and industrial development and its dwindling availability resulting from pollution and resource depletion (Boretti and Rosa, 2019). Escalating water scarcity due to its increasing demand from other sectors threatens the sustainability of irrigated rice production and calls for development of innovative technologies that necessitate less water than conventional flooded rice without experiencing yield losses. There is clearly an urgent need to find ways to grow more rice with less water and fewer inputs. Hence, there is an urgent need to study the effects of different $\mathrm{N}$ management practices on growth, yield and water use efficiency of rice crop under varying moisture regimes.

\section{Material and Methods \\ Details of the study area}

The pot experiment was conducted in polyhouse of the College of Agriculture, Central Agricultural University, Imphal campus during the kharif 2018 and 2019. The geographical position of the polyhouse is as such - Latitude $24^{0} 45^{\prime} \mathrm{N}$, Longitude $93^{0} 54^{\prime} \mathrm{E}$ with an altitude of $774 \mathrm{~m}$ above mean sea level. 245.9 and $236.14 \mathrm{~mm}$ of mean monthly rainfalls were recorded during kharif 2018 and 2019, respectively. Monthly mean temperature ranged from 11.1 to $22.6{ }^{\circ} \mathrm{C}$ and 13.5 to $22.5^{\circ} \mathrm{C}$, respectively during both years of investigation. The soil was clay in texture $(13.6 \%$ sand, $24.3 \%$ silt, and $62.1 \%$ clay), acidic ( $\mathrm{pH} 5.4$ ) and low in available phosphorus $(\mathrm{P})$ with medium in available $\mathrm{N}$ and potassium $(\mathrm{K})$ status.

\section{Treatment details}

The pot experiment was laid out in Factorial Randomized Block Design with 3 levels of Nutrient Management Practices $\left\{\mathrm{N}_{1}: 100 \%\right.$ recommended dose of $\mathrm{N}(\mathrm{RDN})$ throughUrea, $\mathrm{N}_{2}: 66.67 \% \mathrm{RDN}$ through Urea $+33.33 \%$ RDN through FYM, $\mathrm{N}_{3}$ : 
$50 \%$ RDN through Urea $+25 \%$ RDN through FYM $+25 \%$ RDN through Azolla $\}$ and 3 levels of moisture regime $\left\{\mathrm{M}_{1}\right.$ : Continuous flooding $(5 \mathrm{~cm}$ depth); $\mathrm{M}_{2}$ : No standing water (wetting soil just after hairy cracks appear); $\mathrm{M}_{3}: 5 \mathrm{~cm}$ water depth at tillering, panicle initiation and flowering stages which were combined into 9 treatments and replicated thrice. $60 \mathrm{~kg} \mathrm{~N} / \mathrm{ha}$ was the recommended dose of $\mathrm{N}$ in rice.

\section{Pot preparation}

Soil was collected from a farm field for filling the pots. In this case, the field was disturbed by giving one deep ploughing. Then soils were selected from 5 different random spots of the field and they had been evenly mixed together before filling the pots. Pots of dimension $24 \mathrm{~cm} \times 24 \mathrm{~cm} \times 30 \mathrm{~cm}$ each with no single hole on them was used for the experiment. Each pot was filled with measured 10 $\mathrm{kg}$ of pre-collected soil and was kept as according to the experimental design alignment and labelled. As per our nutrient management criteria, $\mathrm{N}_{2}$ and $\mathrm{N}_{3}$ labelled pots needed addition of FYM and both Azolla and FYM respectively other than inorganic nutrients. So, about 15 days before transplantation, those nutrient supplements were added to their respective pots in their mentionable amount. Just before transplanting, the soils in the pots were tilled using any hand tool (khurpi) and was irrigated up to $5 \mathrm{~cm}$ depth, resembling a puddled field condition. Seed was sown on first week of July on nursery bed and then transplanted using 23 days old seedlings. 3 seedlings were transplanted in each pot by keeping them in an equal distance of $10 \mathrm{~cm}$ in triangular spacing from each other.

\section{Nutrient and water management}

Application of both inorganic (NPK) and organic (FYM and Azolla) nutrient sources were done as according to the treatment. FYM and Azolla were applied and incorporated to the soil 15 days before transplanting for the recommended pots $\left(\mathrm{N}_{2}\right.$ and $\mathrm{N}_{3}$ labelled). In case of inorganic fertilizers, half of the $\mathrm{N}$ dose i.e., 60,40 and $30 \mathrm{~kg} / \mathrm{ha}$ for $\mathrm{N}_{1}, \mathrm{~N}_{2}$ and $\mathrm{N}_{3}$ respectively and full of the doses of $\mathrm{P}$ and $\mathrm{K}$ were applied as basal, just before transplanting. The remaining half of $\mathrm{N}$ dose was applied into two equal splits as top-dressing at the time of tillering and panicle initiation. Recommended doses of $\mathrm{P}$ and $\mathrm{K}$ were $40 \mathrm{~kg} \mathrm{P}_{2} \mathrm{O}_{5} / \mathrm{ha}$ and $30 \mathrm{~kg} \mathrm{~K}_{2} \mathrm{O} / \mathrm{ha}$, respectively. Super Phosphate and Muriate of
Potash were the sources of $\mathrm{P}$ and $\mathrm{K}$, respectively. Irrigation was started from the time of transplanting with initially keeping $5 \mathrm{~cm}$ stagnation in all treatments. Specified moisture level as per treatment was followed in the subsequent irrigations. Tap water was used as the source of irrigation.

\section{Intercultural operations}

As the crop was transplanted in pots, there was no serious problem of weeds. Only need-based hand manual weeding was done during the crop season. There were no noticeable pest and disease problems except sheath rot disease infestation, during booting stage of the crop. To control it spray of Carbendazim solution@1 g/L was applied 2 times with one week gap.

\section{Biometric observations}

Plant height, number of leaves and tillers per hill were recorded at 40,80 days after transplanting (DAT) and at harvest. Leaf length and leaf width were also recorded at 40,80 DAT and at harvesting. Flag leaf length measurement was recorded at 80 DAT, and at harvest. At physiological maturity, number of effective tillers per plant was counted. Panicle length, number of spikelets and filled spikelets per panicle were determined before determining the grain yield. Test weight was calculated by using the following equation:

Test weight $=\frac{\text { Weight of filled grain }(\mathrm{g})}{\text { Number of filled grain }} \times 1000$

After drying, grain yield and straw yield from each hill from each pot were taken by using a digital electric balance and expressed in gram (g). Harvest index was calculated with following formula (Donald, 1963):

Harvest index $=\frac{\text { Grain yield per pot }(\mathrm{g})}{\text { Biological yield per pot }(\mathrm{g})} \times 100$

Water Use Efficiency (WUE) was measured by using the formula:

Water Use Efficiency $(W U E)=\frac{\text { Grain yield per pot }(g)}{\text { Biological yield per pot }(g)}$ 


\section{Data analysis}

The experimental data of two consecutive years were pooled and analysed statistically by applying the technique of analysis of variance (Gomez and Gomez, 1984) prescribed for the factorial randomized plot design to test the significant difference among treatments by the $\mathrm{F}$ test and conclusions were drawn at $5 \%$ probability levels. The least significant difference (LSD) at $\alpha=0.05$, was used to compare the treatment means. Significance or non-significance of the variance due to various treatment effects were determined by calculating respective 'F' values. Pearson's correlation was performed to determine the relations between the differential yield attributing parameters implicated in yield of crop. The principal component analysis (PCA) of all the data were performed using $\mathrm{R}$ studio for dimension reduction of data set. PCA is a statistical technique aimed to condense dimensions of multivariate dataset by retaining most of useful value of the input data thereby reducing its dimensions (Karkra et al., 2016). It is commonly used procedure for independent variable assortment and eliminating redundant or highly correlated variables. Stepwise multiple linear regression analysis was accomplished using $\mathrm{R}$ studio to explore the significance and contribution of differential yield attributing parameters in the prediction of crop yield.

\section{Results and Discussion Growth attributes}

$\mathrm{N}$ management practice did not exert any significant influence on plant height at all the stages of observation, except at 40 DAT, in which the markedly improved plant height was registered under 100\% RDN through Urea (Table 1). Application of $50 \%$ RDN through Urea $+25 \%$ RDN through FYM $+25 \%$ RDN through Azolla perceptibly enhanced the number of tillers/hill and leaves/hill at all the stages of observation (Table 1). Inorganic fertilization along with organic supplements might have provided adequate nutrient and created a favourable soil physical condition for crop growth, thus improved growth attributes such as number of tillers and leaves at various growth stages. Oo et al. (2010) and Jeyajothi and Durairaj (2015) also observed that synergistic effects of combined application of chemical fertilizers along with organic nutrient sources like FYM, green leaf manuring and biofertilizers boosted the growth parameters of rice. Leaf length and leaf width remained statistically unaffected due to different nutrient management practices (Table 2). The significantly highest flag leaf length was recorded in all the crop growth stages under $100 \% \mathrm{RDN}$ through Urea (Table 2). This could be attributed to the relative quicker and easier nutrient supplying capacity of inorganic fertilizers over organic nutrient sources to plants.

The tallest plant was recorded under continuous flooding in all the stages of observation, which was statistically superior among all moisture regimes (Table 1). However, the significantly highest number of tillers/hill was registered under $5 \mathrm{~cm}$ water depth at tillering, panicle initiation and flowering stages during the entire periodic assessment (Table 2). Growth attributes namely number of leaves/hill (Table 1), leaf length and leaf width (Table 2) did not vary statistically under different moisture regimes. Whereas, a conspicuous improvement in flag leaf length was noted under continuous flooding of rice crop at various stages of observation (Table 2). Alternate wetting and drying irrigation along with maintenance of flooding at the critical stages of crop growth renders a favourable situation of better root and shoot growth of rice (Zhang et al., 2009).

On critical analysis, it was noted that treatment combinations of $\mathrm{N}$ management practice and moisture regime had a marked impact on the growth attributes of rice, except leaf length and leaf width (Table 1 and 2). Application of 100\% RDN through Urea under continuous flooding led to tangible enhancement in plant height, which superior among all treatment combinations. However, the highest number of tillers/hill and number of leaves/hill were found under $50 \%$ RDN through Urea $+25 \%$ RDN through FYM $+25 \%$ RDN through Azolla registered under $5 \mathrm{~cm}$ water depth at tillering, panicle initiation and flowering stages. Flag length was significantly improved due to the application of $100 \%$ RDN through Urea under no flooding, which was statistically at par with $50 \%$ RDN through Urea $+25 \%$ RDN through FYM $+25 \%$ RDN through Azolla registered under $5 \mathrm{~cm}$ water depth at tillering, panicle initiation and flowering stages at all the stages of record. Adequate $\mathrm{N}$ availability during active growth stages 


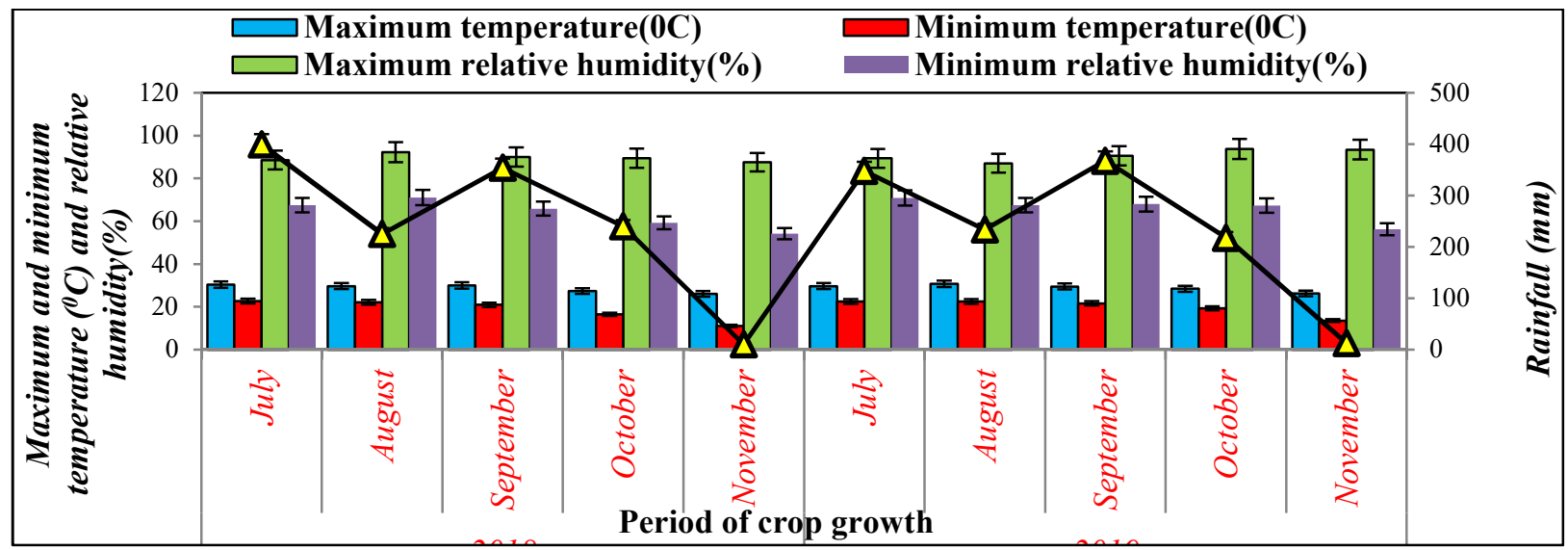

Figure 1: Meteorological observation of the pot experiment of rice during 2018-19

Table 1: Effect of different $N$ management practices and moisture regimes on periodic plant height, number of tillers and leaves per hill of rice

\begin{tabular}{|c|c|c|c|c|c|c|c|c|c|}
\hline \multirow[t]{2}{*}{ Treatments } & \multicolumn{3}{|c|}{ Plant height $(\mathrm{cm})$} & \multicolumn{3}{|c|}{ Number of tillers/hill } & \multicolumn{3}{|c|}{ Number of leaves/hill } \\
\hline & 40 DAT & 80 DAT & $\begin{array}{l}\text { At } \\
\text { harvest }\end{array}$ & $\begin{array}{l}40 \\
\text { DAT }\end{array}$ & $\begin{array}{l}80 \\
\text { DAT }\end{array}$ & $\begin{array}{l}\text { At } \\
\text { harvest }\end{array}$ & $\begin{array}{l}40 \\
\text { DAT }\end{array}$ & $\begin{array}{l}80 \\
\text { DAT }\end{array}$ & $\begin{array}{l}\text { At } \\
\text { harvest }\end{array}$ \\
\hline \multicolumn{10}{|c|}{ Nitrogen management practices $(\mathrm{N})$} \\
\hline $\mathbf{N}_{1}$ & 110.00 & 117.00 & 114.74 & 2.04 & 2.04 & 2.04 & 10.22 & 8.48 & 6.41 \\
\hline $\mathbf{N}_{2}$ & 104.37 & 116.48 & 114.70 & 2.19 & 2.22 & 2.22 & 9.78 & 8.85 & 6.59 \\
\hline $\mathbf{N}_{3}$ & 105.01 & 117.00 & 115.30 & 2.30 & 2.52 & 2.52 & 12.15 & 10.04 & 7.39 \\
\hline S.E(d) \pm & 0.61 & 0.56 & 0.87 & 0.11 & 0.10 & 0.10 & 0.39 & 0.52 & 0.37 \\
\hline $\operatorname{LSD}_{(\mathrm{P}=0.05)}$ & 1.29 & NS & NS & 0.23 & 0.20 & 0.20 & 0.82 & 1.11 & 0.79 \\
\hline \multicolumn{10}{|c|}{ Moisture regimes (M) } \\
\hline $\mathbf{M}_{1}$ & 107.46 & 119.11 & 117.04 & 2.11 & 2.15 & 2.15 & 10.52 & 8.81 & 6.85 \\
\hline $\mathbf{M}_{2}$ & 106.15 & 117.96 & 116.00 & 2.11 & 2.22 & 2.22 & 10.59 & 8.78 & 6.26 \\
\hline $\mathbf{M}_{3}$ & 105.78 & 113.41 & 111.70 & 2.30 & 2.41 & 2.41 & 11.04 & 9.78 & 6.96 \\
\hline 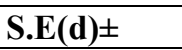 & 0.61 & 0.56 & 0.87 & 0.11 & 0.10 & 0.10 & 0.39 & 0.52 & 0.37 \\
\hline $\mathbf{L S D}_{(\mathrm{P}=\mathbf{0 . 0 5})}$ & 1.29 & 1.19 & 1.84 & NS & 0.20 & 0.20 & NS & NS & NS \\
\hline \multicolumn{10}{|c|}{ Interaction $(\mathrm{N} \times \mathrm{M})$} \\
\hline $\mathbf{N}_{1} \mathbf{M}_{1}$ & 114.22 & 122.56 & 118.89 & 2.11 & 2.11 & 2.11 & 11.00 & 8.78 & 6.67 \\
\hline $\mathbf{N}_{1} \mathbf{M}_{2}$ & 107.78 & 118.11 & 116.44 & 2.00 & 2.00 & 2.00 & 10.22 & 8.11 & 6.11 \\
\hline $\mathbf{N}_{1} \mathbf{M}_{3}$ & 108.00 & 110.33 & 108.89 & 2.00 & 2.00 & 2.00 & 9.44 & 8.56 & 6.44 \\
\hline $\mathbf{N}_{2} \mathrm{M}_{1}$ & 104.56 & 117.00 & 115.89 & 2.11 & 2.22 & 2.22 & 10.33 & 9.00 & 7.11 \\
\hline $\mathbf{N}_{2} \mathbf{M}_{2}$ & 104.89 & 117.11 & 114.89 & 2.22 & 2.22 & 2.22 & 9.00 & 8.56 & 5.89 \\
\hline $\mathbf{N}_{2} \mathbf{M}_{3}$ & 103.67 & 115.33 & 113.33 & 2.22 & 2.22 & 2.22 & 10.00 & 9.00 & 6.78 \\
\hline $\mathbf{N}_{3} \mathbf{M}_{1}$ & 103.59 & 117.78 & 116.33 & 2.11 & 2.11 & 2.11 & 10.22 & 8.67 & 6.78 \\
\hline $\mathbf{N}_{3} \mathbf{M}_{2}$ & 105.78 & 118.67 & 116.67 & 2.11 & 2.44 & 2.44 & 12.56 & 9.67 & 6.78 \\
\hline $\mathbf{N}_{3} \mathbf{M}_{3}$ & 105.67 & 114.56 & 112.89 & 2.67 & 3.00 & 3.00 & 13.67 & 11.78 & 7.67 \\
\hline S.E(d) \pm & 1.05 & 0.97 & 1.50 & 0.19 & 0.17 & 0.17 & 0.67 & 0.91 & 0.63 \\
\hline $\operatorname{LSD}_{(\mathrm{P}=0.05)}$ & 2.24 & 2.07 & 3.18 & 0.40 & 0.37 & 0.37 & 1.43 & 1.92 & 1.34 \\
\hline
\end{tabular}

due to integrated $\mathrm{N}$ supplementation from both Yield attributes

organic and inorganic coupled with adequate water Different $\mathrm{N}$ management practices had a noticeable availability could have resulted in marked impact on yield attributes of rice, except spikelets/ improvement in growth and development of rice. panicle, filled grains/panicle and panicle length 
Table 2: Effect of different $N$ management practices and moisture regimes on periodic leaf length, width and flag leaf length of rice

\begin{tabular}{|c|c|c|c|c|c|c|c|c|c|}
\hline \multirow[t]{2}{*}{ Treatments } & \multirow{2}{*}{$\begin{array}{l}\begin{array}{l}\text { Leaf } \\
\text { length } \\
\text { (cm) }\end{array} \\
40 \text { DAT }\end{array}$} & \multirow{2}{*}{\begin{tabular}{|l|}
$\begin{array}{l}\text { Leaf } \\
\text { width } \\
\text { (cm) }\end{array}$ \\
80 DAT
\end{tabular}} & \multirow{2}{*}{$\begin{array}{l}\begin{array}{l}\text { Flag leaf } \\
\text { length }(\mathrm{cm})\end{array} \\
\text { At harvest }\end{array}$} & \multirow[b]{2}{*}{$\begin{array}{l}40 \\
\text { DAT }\end{array}$} & \multirow[b]{2}{*}{$\begin{array}{l}80 \\
\text { DAT }\end{array}$} & \multirow[b]{2}{*}{$\begin{array}{l}\text { At } \\
\text { harvest }\end{array}$} & \multirow[b]{2}{*}{$\begin{array}{l}\text { 60 } \\
\text { DAT }\end{array}$} & \multirow[b]{2}{*}{$\begin{array}{l}\text { 100 } \\
\text { DAT }\end{array}$} & \multirow[b]{2}{*}{$\begin{array}{l}\text { At } \\
\text { harvest }\end{array}$} \\
\hline & & & & & & & & & \\
\hline \multicolumn{10}{|c|}{ Nitrogen management practices $(\mathrm{N})$} \\
\hline $\mathrm{N}_{1}$ & 65.94 & 75.62 & 68.70 & 1.69 & 1.88 & 1.78 & 38.90 & 43.47 & 41.20 \\
\hline $\mathrm{N}_{2}$ & 65.68 & 74.73 & 68.42 & 1.68 & 1.88 & 1.78 & 33.51 & 38.84 & 35.08 \\
\hline $\mathrm{N}_{3}$ & 65.31 & 75.14 & 68.54 & 1.68 & 1.91 & 1.79 & 32.51 & 37.23 & 34.22 \\
\hline S.E(d) \pm & 0.52 & 0.51 & 0.15 & 0.01 & 0.02 & 0.01 & 1.05 & 0.80 & 1.25 \\
\hline $\mathrm{LSD}_{(\mathrm{P}=0.05)}$ & NS & NS & NS & NS & NS & NS & 2.23 & 1.69 & 2.65 \\
\hline \multicolumn{10}{|c|}{ Moisture regimes (M) } \\
\hline $\mathrm{M}_{1}$ & 65.58 & 75.27 & 68.71 & 1.69 & 1.89 & 1.79 & 37.01 & 41.63 & 39.35 \\
\hline $\mathrm{M}_{2}$ & 66.07 & 74.96 & 68.42 & 1.67 & 1.88 & 1.78 & 34.19 & 38.35 & 36.53 \\
\hline $\mathrm{M}_{3}$ & 65.27 & 75.25 & 68.54 & 1.69 & 1.90 & 1.78 & 33.70 & 39.56 & 34.62 \\
\hline S.E(d) \pm & 0.52 & 0.51 & 0.15 & 0.01 & 0.02 & 0.01 & 1.05 & 0.80 & 1.25 \\
\hline $\mathrm{LSD}_{(\mathrm{P}=0.05)}$ & NS & NS & $\mathrm{NS}$ & 0.03 & NS & NS & 2.23 & 1.69 & 2.65 \\
\hline \multicolumn{10}{|c|}{ Interaction $(\mathbf{N} \times \mathbf{M})$} \\
\hline $\mathrm{N}_{1} \mathrm{M}_{1}$ & 66.01 & 76.22 & 68.89 & 1.68 & 1.89 & 1.8 & 41.13 & 45.57 & 43.54 \\
\hline $\mathrm{N}_{1} \mathrm{M}_{2}$ & 65.96 & 75.41 & 68.85 & 1.67 & 1.87 & 1.77 & 40.44 & 45.48 & 42.78 \\
\hline $\mathrm{N}_{1} \mathrm{M}_{3}$ & 65.86 & 75.22 & 68.37 & 1.71 & 1.87 & 1.77 & 35.11 & 39.35 & 37.28 \\
\hline $\mathrm{N}_{2} \mathrm{M}_{1}$ & 65.85 & 74.59 & 68.41 & 1.68 & 1.88 & 1.78 & 35.11 & 39.44 & 35.02 \\
\hline $\mathrm{N}_{2} \mathrm{M}_{2}$ & 64.7 & 74.89 & 68.41 & 1.67 & 1.87 & 1.76 & 30.91 & 38.74 & 33.43 \\
\hline $\mathrm{N}_{2} \mathrm{M}_{3}$ & 66.48 & 74.7 & 68.44 & 1.68 & 1.88 & 1.79 & 34.5 & 38.33 & 36.78 \\
\hline $\mathrm{N}_{3} \mathrm{M}_{1}$ & 65.78 & 74.78 & 68.33 & 1.67 & 1.88 & 1.77 & 27.63 & 31.24 & 30.04 \\
\hline $\mathrm{N}_{3} \mathrm{M}_{2}$ & 64.11 & 74.93 & 68.44 & 1.67 & 1.93 & 1.79 & 30.89 & 39.89 & 31.56 \\
\hline $\mathrm{N}_{3} \mathrm{M}_{3}$ & 66.04 & 75.71 & 68.86 & 1.71 & 1.92 & 1.82 & 39 & 40.57 & 41.07 \\
\hline 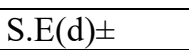 & 0.91 & 0.89 & 0.26 & 0.02 & 0.03 & 0.02 & 1.82 & 1.38 & 2.17 \\
\hline $\operatorname{LSD}_{(\mathrm{P}=0.05)}$ & NS & NS & NS & NS & NS & NS & 3.86 & 2.92 & 4.60 \\
\hline
\end{tabular}

(Table 3). The maximum values of effective Treatment no standing water recorded the tillers/hill and test weight were registered under statistically the highest number of effective $50 \%$ RDN through Urea $+25 \%$ RDN through FYM tillers/hill. Treatment continuous flooding led to $+25 \%$ RDN through Azolla, which was marked improvement in number of significantly the highest among all the treatments. spikelets/panicle and filled grains/panicle, which Enhanced soil aggregation, improved nutrient was statistically at par with no standing water. A availability and soil microbial activity under favourable growing and nutrient supply integrated nutrient management might have created environment under continuous flooding might have a favourable soil environment, which further better increased number of spikelets and partitioning of the uptake of nutrients, and consequently leading to dry matters at grain filling stage results in higher prolific vegetative growth of plants and higher dry filled grains in panicle. Ramakrishna et al. (2007) matter accumulation (Nataraja et al., 2021). reported that continuous water submergence Adequate and efficient supply of plant nutrients due markedly enhanced yield attributes (including to the combined application of urea along with number of panicles, grains/panicle, 1,000-grain FYM and Azolla might have beneficial effect on weight) of rice. However, a safe alternate wetting yield attributes like effective tillers per hill and test and drying irrigation situation has been observed to weight of rice. Yield attributes of rice, except, be equally capable of providing congenial panicle length and test weight, responded environment by facilitating crop to access moisture significantly to varying moisture regimes (Table 3). from subsurface levels, which remain saturated and 
Table 3: Effect of different $\mathrm{N}$ management practices and moisture regimes on yield attributes, yields and water use efficiency of rice

\begin{tabular}{|c|c|c|c|c|c|c|c|c|c|}
\hline Treatments & $\begin{array}{l}\text { Effective } \\
\text { Tillers/hill }\end{array}$ & $\begin{array}{l}\text { Spikelets/ } \\
\text { panicle }\end{array}$ & $\begin{array}{l}\text { Filled } \\
\text { grains/ } \\
\text { panicle }\end{array}$ & $\begin{array}{l}\text { Panicle } \\
\text { length } \\
(\mathrm{cm})\end{array}$ & $\begin{array}{l}\text { Test } \\
\text { Weight } \\
\text { (g) }\end{array}$ & $\begin{array}{l}\text { Grain } \\
\text { Yield } \\
\text { (g/hill) }\end{array}$ & $\begin{array}{l}\text { Straw } \\
\text { Yield } \\
\text { (g/hill) }\end{array}$ & $\begin{array}{l}\text { Harvest } \\
\text { Index } \\
(\%)\end{array}$ & WUE (g/l) \\
\hline \multicolumn{10}{|c|}{ Nitrogen management practices $(\mathbf{N})$} \\
\hline $\mathrm{N}_{1}$ & 2.04 & 144.20 & 128.96 & 22.27 & 26.18 & 6.35 & 4.79 & 57.09 & 1.28 \\
\hline $\mathrm{N}_{2}$ & 2.04 & 148.00 & 132.73 & 21.88 & 26.28 & 6.32 & 5.47 & 55.99 & 1.33 \\
\hline $\mathrm{N}_{3}$ & 2.48 & 147.98 & 128.55 & 22.24 & 28.08 & 7.55 & 5.87 & 57.57 & 1.67 \\
\hline S.E(d) \pm & 0.12 & 3.75 & 2.86 & 0.30 & 0.58 & 0.19 & 0.24 & 1.60 & 0.07 \\
\hline $\mathrm{LSD}_{(\mathrm{P}=0.05)}$ & 0.25 & $\mathrm{NS}$ & $\mathrm{NS}$ & $\mathrm{NS}$ & 1.24 & 0.41 & 0.50 & $\mathrm{NS}$ & 0.15 \\
\hline \multicolumn{10}{|c|}{ Moisture regimes (M) } \\
\hline $\mathrm{M}_{1}$ & 2.11 & 150.47 & 133.98 & 22.29 & 26.80 & 6.67 & 5.27 & 56.15 & 0.91 \\
\hline $\mathrm{M}_{2}$ & 2.07 & 149.70 & 132.76 & 22.06 & 26.67 & 6.52 & 4.95 & 58.87 & 1.64 \\
\hline $\mathrm{M}_{3}$ & 2.37 & 140.01 & 123.51 & 22.04 & 27.07 & 7.01 & 5.90 & 55.63 & 1.74 \\
\hline S.E(d)士 & 0.12 & 3.75 & 2.86 & 0.30 & 0.58 & 0.19 & 0.24 & 1.60 & 0.07 \\
\hline $\operatorname{LSD}_{(\mathrm{P}=0.05)}$ & 0.25 & 7.95 & 6.06 & NS & $\mathrm{NS}$ & 0.41 & 0.50 & NS & 0.15 \\
\hline \multicolumn{10}{|c|}{ Interaction $(\mathrm{N} \times \mathbf{M})$} \\
\hline $\mathrm{N}_{1} \mathrm{M}_{1}$ & 2.11 & 151.39 & 136.33 & 23.13 & 27.06 & 6.68 & 4.60 & 59.87 & 0.89 \\
\hline $\mathrm{N}_{1} \mathrm{M}_{2}$ & 2.00 & 153.33 & 138.11 & 22.11 & 26.39 & 6.62 & 3.98 & 62.94 & 1.62 \\
\hline $\mathrm{N}_{1} \mathrm{M}_{3}$ & 2.00 & 127.89 & 112.44 & 21.56 & 25.08 & 5.75 & 5.78 & 48.48 & 1.33 \\
\hline $\mathrm{N}_{2} \mathrm{M}_{1}$ & 2.11 & 147.48 & 134.89 & 21.54 & 26.99 & 6.67 & 5.48 & 55.27 & 0.93 \\
\hline $\mathrm{N}_{2} \mathrm{M}_{2}$ & 1.78 & 148.83 & 134.17 & 22.22 & 25.73 & 5.46 & 5.43 & 53.81 & 1.31 \\
\hline $\mathrm{N}_{2} \mathrm{M}_{3}$ & 2.22 & 147.69 & 129.15 & 21.89 & 26.13 & 6.82 & 5.50 & 58.88 & 1.74 \\
\hline $\mathrm{N}_{3} \mathrm{M}_{1}$ & 2.11 & 152.54 & 130.70 & 22.20 & 26.34 & 6.67 & 5.74 & 53.31 & 0.91 \\
\hline $\mathrm{N}_{3} \mathrm{M}_{2}$ & 2.44 & 146.93 & 126.00 & 21.85 & 27.90 & 7.49 & 5.43 & 59.87 & 1.97 \\
\hline $\mathrm{N}_{3} \mathrm{M}_{3}$ & 2.89 & 144.46 & 128.94 & 22.67 & 29.99 & 8.48 & 6.42 & 59.53 & 2.13 \\
\hline S.E(d)士 & 0.21 & 6.49 & 4.95 & 0.51 & 1.01 & 0.34 & 0.41 & 2.76 & 0.12 \\
\hline $\operatorname{LSD}_{(\mathrm{P}=0.05)}$ & 0.45 & 13.77 & 10.50 & 1.09 & 2.14 & 0.71 & NS & 5.86 & 0.26 \\
\hline
\end{tabular}


Table 4: Pearson's correlation coefficient ( $r$ ) matrix between the different soil chemicals, biological properties and with grain yield of rice

\begin{tabular}{|c|c|c|c|c|c|c|c|c|c|c|c|c|}
\hline 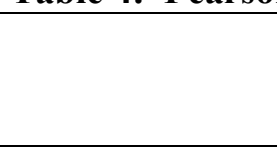 & $\begin{array}{l}\text { Plant } \\
\text { height }\end{array}$ & $\begin{array}{l}\text { Number } \\
\text { of } \\
\text { tillers/hill }\end{array}$ & $\begin{array}{l}\text { Number } \\
\text { of } \\
\text { leaves/hill }\end{array}$ & $\begin{array}{l}\text { Leaf } \\
\text { length }\end{array}$ & $\begin{array}{l}\text { Leaf } \\
\text { width }\end{array}$ & $\begin{array}{l}\text { Flag } \\
\text { leaf } \\
\text { length }\end{array}$ & $\begin{array}{l}\text { Effective } \\
\text { Tillers/hill }\end{array}$ & $\begin{array}{l}\text { Spikelets/ } \\
\text { panicle }\end{array}$ & $\begin{array}{l}\text { Filled } \\
\text { grains/ } \\
\text { panicle }\end{array}$ & $\begin{array}{l}\text { Panicle } \\
\text { length }\end{array}$ & $\begin{array}{l}\text { Test } \\
\text { Weight }\end{array}$ & $\begin{array}{l}\text { Grain } \\
\text { Yield }\end{array}$ \\
\hline Plant height & 1.000 & -0.111 & -0.072 & 0.330 & 0.073 & 0.036 & -0.091 & 0.874 & 0.816 & 0.478 & 0.207 & 0.150 \\
\hline $\begin{array}{l}\text { Number } \\
\text { tillers/hill }\end{array}$ & -0.111 & 1.000 & 0.739 & 0.302 & 0.769 & 0.062 & 0.893 & -0.010 & -0.023 & 0.292 & 0.905 & 0.815 \\
\hline $\begin{array}{l}\text { Number } \\
\text { leaves/hill }\end{array}$ & -0.072 & 0.739 & 1.000 & 0.183 & 0.815 & 0.065 & 0.866 & -0.068 & -0.097 & 0.140 & 0.786 & 0.833 \\
\hline Leaf length & 0.330 & 0.302 & 0.183 & 1.000 & 0.584 & 0.887 & 0.370 & 0.309 & 0.449 & 0.737 & 0.532 & 0.464 \\
\hline Leaf width & 0.073 & 0.769 & 0.815 & 0.584 & 1.000 & 0.445 & 0.884 & 0.032 & 0.032 & 0.517 & 0.854 & 0.864 \\
\hline $\begin{array}{ll}\text { Flag } & \text { leaf } \\
\text { length }\end{array}$ & 0.036 & 0.062 & 0.065 & 0.887 & 0.445 & 1.000 & 0.160 & 0.011 & 0.241 & 0.523 & 0.229 & 0.199 \\
\hline $\begin{array}{l}\text { Effective } \\
\text { Tillers/hill }\end{array}$ & -0.091 & 0.893 & 0.866 & 0.370 & 0.884 & 0.160 & 1.000 & -0.049 & -0.134 & 0.258 & 0.913 & 0.959 \\
\hline $\begin{array}{l}\text { Spikelets/ } \\
\text { panicle }\end{array}$ & 0.874 & -0.010 & -0.068 & 0.309 & 0.032 & 0.011 & -0.049 & 1.000 & 0.918 & 0.444 & 0.211 & 0.199 \\
\hline $\begin{array}{l}\text { Filled grains/ } \\
\text { panicle }\end{array}$ & 0.816 & -0.023 & -0.097 & 0.449 & 0.032 & 0.241 & -0.134 & 0.918 & 1.000 & 0.457 & 0.198 & 0.099 \\
\hline Panicle length & 0.478 & 0.292 & 0.140 & 0.737 & 0.517 & 0.523 & 0.258 & 0.444 & 0.457 & 1.000 & 0.439 & 0.313 \\
\hline Test Weight & 0.207 & 0.905 & 0.786 & 0.532 & 0.854 & 0.229 & 0.913 & 0.211 & 0.198 & 0.439 & 1.000 & 0.934 \\
\hline Grain Yield & 0.150 & 0.815 & 0.833 & 0.464 & 0.864 & 0.199 & 0.959 & 0.199 & 0.099 & 0.313 & 0.934 & 1.000 \\
\hline
\end{tabular}

Boldfaced italics and boldface values indicate that correlation is positive and highly significant at $p<0.01 \%$ and $p<0.05 \%$ level of probability

( 2 tailed). The correlation coefficient $(r)$ values correspond directly to the colour codes from green to yellow and red, respectively. 
improving nutrient availability through better $\mathrm{N}$ mineralization (Lu et al., 2000; Ceesay et al., 2006; Pandey et al., 2010).

Various combinations of $\mathrm{N}$ management practice and moisture regime induced a significant influence on yield attributes of rice (Table 3). Application of $50 \%$ RDN through Urea $+25 \%$ RDN through FYM $+25 \%$ RDN through Azolla under $5 \mathrm{~cm}$ depth at tillering, panicle initiation and flowering stages resulted in the highest number of effective tillers/hill which was significantly better than rest of treatment combinations, except $50 \%$ RDN through Urea $+25 \%$ RDN through FYM $+25 \%$ RDN through Azolla under no standing water. However, a conspicuous increase in number of spikelets/panicle and filled spikelets/panicle was recorded due to application of $66.67 \% \mathrm{RDN}$ through Urea $+33.33 \%$ RDN through FYM under continuous flooding. The highest panicle length was registered under $100 \%$ RDN through Urea under continuous flooding, which statistically equivalent to $100 \%$ RDN through Urea under no flooding, $66.67 \%$ RDN through Urea $+33.33 \%$ RDN through FYM under no standing water, 50\% RDN through Urea $+25 \%$ RDN through FYM + 25\% through Azolla under continuous flooding and $5 \mathrm{~cm}$ depth at tillering, panicle initiation and flowering stages. Application of 50\% RDN through Urea $+25 \%$ RDN through FYM $+25 \%$ through Azolla under $5 \mathrm{~cm}$ at tillering, panicle initiation and flowering stages gave the maximum value of test weight, which was significantly higher than the rest of treatment combinations, $50 \% \mathrm{RDN}$ through Urea $+25 \%$ RDN through FYM $+25 \%$ through Azolla under no standing water. The synergism between integrated approach of $\mathrm{N}$ supply and standing water at critical stages could have caused better nutrient availability and its partitioning, thus significant improvement in yield attributes of rice.

\section{Yields and water use efficiency}

Grain and straw yield, and water use efficiency of rice were statistically influenced by different $\mathrm{N}$ management practices and moisture regimes (Table $3)$. However, treatment effect on harvest index was non-significant. Application of 50\% RDN through Urea $+25 \%$ RDN through FYM $+25 \%$ through Azolla significantly bettered both the grain and straw yield over $100 \%$ RDN through Urea. The gain in grain and straw yield due this treatment was in the tune of $18.90 \%$ and $22.55 \%$ over $100 \% \mathrm{RDN}$ through Urea. Being a constituent of amino acids, nucleotides, nucleic acid, a number of coenzyme and growth promoters, $\mathrm{N}$ promotes cell elongation, cell enlargement and cell division. These activities in turn activate meristematic tissues which remain functional for longer periods resulting in better expression of yield and yield attributes converting more solar energy to productive energy. Controlled release of nutrients, including $\mathrm{N}$ through mineralisation of organic manures under integrated nutrient management practices facilitate better crop growth (Meshram et al., 2018) and improved yield attributes namely number of effective tillers/hill and test weight, which ultimately translate into higher yield of rice. Similarly, the highest water use efficiency was registered under 50\% RDN through Urea $+25 \%$ RDN through FYM $+25 \%$ through Azolla. Efficient plant nutrition is a good strategy to enhance yield, water use efficiency and productivity in crop plants (Waraich et al., 2011). Limited researchers have also opined that the integrated use of organic and inorganic nutrient sources in rice cultivation has the advantage in terms of higher yield and water use efficiency of the rice (Singh and Shivay, 2003).

Among different moisture regimes, the maximum value of grain yield was recorded under $5 \mathrm{~cm}$ depth at tillering, panicle initiation and flowering stages, which was statistically at par with continuous flooding. Similarly, treatment $5 \mathrm{~cm}$ depth at tillering, panicle initiation and flowering stages gave the statistically highest value of straw yield among all treatments. However, the harvest index remained significantly unaffected under varying moisture regimes. The highest value of water use efficiency was noted under maintenance of $5 \mathrm{~cm}$ depth at tillering, panicle initiation and flowering stages. Alternate wetting and drying irrigation practice, including flooding only in certain growth stages consumes less quantity of water than conventional method of flooding throughout the season due to reduction in percolation losses. On the other hand, meeting the moisture demand under recommended submergence at specific growth stages provide favourable growing environment with better nutrient availability, thus leading to better yield and WUE as compared to conventional methods (Pandey et al., 2010; Xu et al., 2020). 
Table 5: Results of PCA of soil chemical properties and nutrient status of rice

\begin{tabular}{|l|l|l|l|l|}
\hline Principal components & PC 1 & PC 2 & PC 3 \\
\hline \multirow{3}{*}{ Initial Eigenvalues } & Total & 5.07 & 3.27 & 1.58 \\
\cline { 2 - 5 } & \% of Variance & 46.11 & 29.70 & 14.33 \\
\cline { 2 - 5 } & Cumulative \% & 46.11 & 75.80 & 90.13 \\
\hline \multirow{2}{*}{$\begin{array}{l}\text { Sotation Squared Loadings } \\
\text { Eigen vectors }\end{array}$} & Total & 4.38 & 3.01 & 2.53 \\
\cline { 2 - 5 } & \% of Variance & 39.80 & 27.36 & 22.97 \\
\cline { 2 - 5 } & Cumulative \% & 39.80 & 67.16 & 90.13 \\
\hline Plant height & \multicolumn{2}{|l|}{ actor loadings } & \multicolumn{1}{|c|}{} \\
\cline { 2 - 5 } & PC 1 & PC 2 & PC 3 \\
\hline Number of tillers/hill & -0.040 & 0.922 & 0.101 \\
\hline Number of leaves/hill & $\mathbf{0 . 9 6 0}$ & -0.017 & 0.045 \\
\hline Leaf length & $\mathbf{0 . 8 6 3}$ & -0.069 & 0.088 \\
\hline Leaf width & 0.215 & -0.545 & 0.795 \\
\hline Flag leaf length & 0.696 & -0.551 & 0.071 \\
\hline Effective Tillers/hill & -0.705 & 0.297 & 0.576 \\
\hline Spikelets/panicle & $\mathbf{0 . 9 5 0}$ & -0.053 & 0.106 \\
\hline Filled grains/panicle & -0.008 & 0.991 & -0.009 \\
\hline Panicle length & -0.032 & 0.932 & 0.081 \\
\hline Test weight & 0.215 & 0.453 & 0.826 \\
\hline
\end{tabular}

Extraction Method: Principal Component Analysis,

Rotation Method: Varimax with Kaiser Normalization.

${ }^{a}$ Rotation converged in 5 iterations and

${ }^{b}$ Boldfaced factor loadings are considered highly weighted

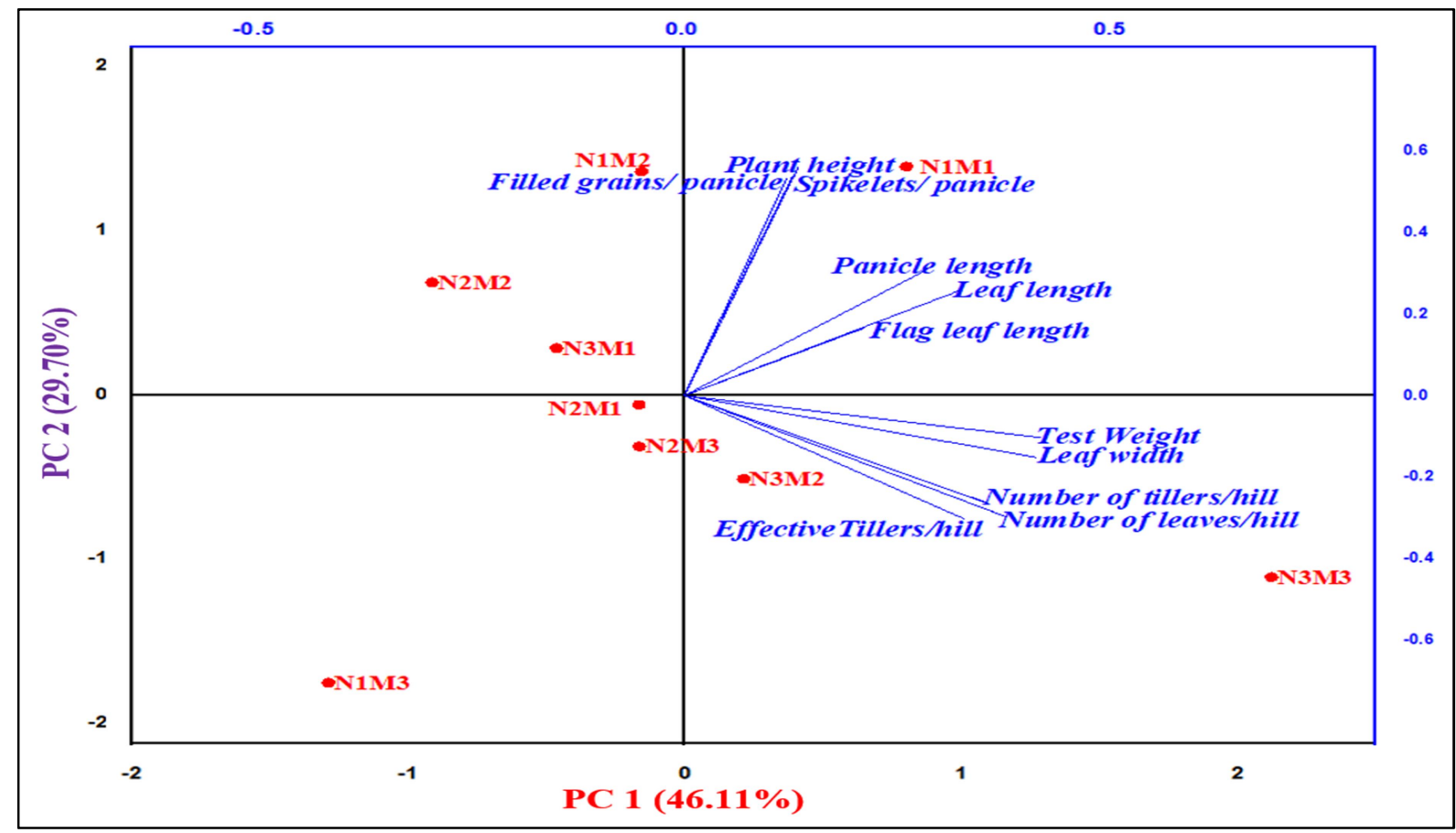

Figure 2: Two dimensional graphical biplot formed by PC 1 and 2 of growth and yield attributing characters of rice 
Zhang et al. (2009) observed that a moderate wetting and drying regime could augment root growth which aids other physiological processes and upshot in higher grain yield and WUE in rice. Various combinations of $\mathrm{N}$ management practice and moisture regimes rendered significant effect on grain yield, and water use efficiency, except straw yield of rice (Table 3). Application of $50 \% \mathrm{RDN}$ through Urea $+25 \%$ RDN through FYM $+25 \%$ through Azolla under $5 \mathrm{~cm}$ depth at tillering, panicle initiation and flowering stages led to marked enhancement of grain yield of rice which was $26.95 \%$ higher than that of $100 \%$ RDN through Urea under continuous flooding. The highest harvest index was recorded under both $100 \% \mathrm{RDN}$ through Urea under continuous flooding and 50\% RDN through Urea $+25 \%$ RDN through FYM + 25\% through Azolla under no standing water, which was statistically at par with $50 \%$ RDN through Urea $+25 \%$ RDN through FYM $+25 \%$ through Azolla under $5 \mathrm{~cm}$ depth at tillering, panicle initiation and flowering stages and $66.67 \%$ RDN through Urea $+33 \%$ RDN through FYM under 5 $\mathrm{cm}$ depth at tillering, panicle initiation and flowering stages. The treatment combination 50\% RDN through Urea $+25 \%$ RDN through FYM + 25\% through Azolla under $5 \mathrm{~cm}$ depth at tillering, panicle initiation and flowering stages showed to have an edge in term of water use efficiency among all the treatment combinations. Higher yield level rice under the combination of $50 \%$ RDN through Urea $+25 \%$ RDN through FYM $+25 \%$ through Azolla under $5 \mathrm{~cm}$ depth at tillering, panicle initiation and flowering stages could be attributed to improved growth and yield attributes under integrated $\mathrm{N}$ management practice with standing water at critical stages of rice. Further, the higher production level with lesser water consumption caused enhancement in its WUE under this treatment combination.

\section{Correlation study between yield attributing} characters of rice and with crop yield

The Pearson's correlation coefficients analysis was performed to evaluate the relationships between the diverse growth and yield attributing characters of rice and its grain yield (Table 4). In general, there existed positive correlation amongst the yield attributing characters of rice and with crop yield. However, few selected parameters revealed the existence of non-significant and negative correlation between them and with crop yield. Specifically, there was an existence of highly and significant positive correlation of plant height with spikelets/panicle $(r=0.874, p<0.01)$ and filled grains/ panicle $(r=0.816, p<0.01)$ but it showed non-significant negative correlation with number of tillers/hill $(\mathrm{r}=-0.111)$, number of leaves/hill (0.072) and effective tillers/hill $(\mathrm{r}=-0.091)$. The number of tillers/hill was found to correlate (highly and significant positive correlation) with number of leaves/hill $(\mathrm{r}=0.739, \mathrm{p}<0.01)$, Leaf width $(\mathrm{r}=$ $0.769, \mathrm{p}<0.01)$, effective tillers/hill $(\mathrm{r}=0.893, \mathrm{p}<$ $0.01)$, test weight $(\mathrm{r}=0.905, \mathrm{p}<0.01)$ and grain yield $(\mathrm{g} / \mathrm{hill})(\mathrm{r}=0.815, \mathrm{p}<0.01)$ while it revealed non-significant negative correlation with spikelets/ panicle $(r=-0.010)$ and filled grains/ panicle $(r=$ 0.023). Similarly, the number of leaves/hill exhibited a significant positive correlation correlated with leaf width $(\mathrm{r}=0.815, \mathrm{p}<0.05)$, effective tillers/hill $(r=0.866, p<0.01)$, test weight $(r=0.786, p<0.01)$, and grain yield $(r=$ $0.833, \mathrm{p}<0.01)$, however it was found to correlative negatively with spikelets/ panicle $(r=$ 0.068 ) and filled grains/ panicle $(\mathrm{r}=-0.097)$. There existed a significant positive correlation of leaf length with leaf width $(\mathrm{r}=0.584, \mathrm{p}<0.05)$, Flag leaf length $(r=0.887, p<0.01)$, panicle length $(r=0.737, p<0.01)$ and test weight $(r=0.532, p<$ 0.01 ). Also, effective tillers/hill revealed a highly and significant positive correlation with test weight $(r=0.913, p<0.01)$ and grain yield $(r=0.913, p<$ $0.001)$ and grain yield $(r=0.959, p<0.001)$ but it exhibited a non-significant negative correlation with spikelets/panicle $(r=-0.049)$ and Filled grains/ panicle $(r=-0.134)$. While, pikelets/ panicle exhibited a highly and significant positive correlation with filled grains/ panicle $(r=0.918, p$ $<0.01$ ) while it revealed non-significant negative correlation with spikelets/ panicle $(r=-0.010)$. And finally, test weight showed the existence of a highly and significant positive correlation with grain yield $(r=0.934, p<0.01)$. Similar findings were also reported by Jat et al. (2016) and (2020).

\section{Principal component analysis}

The results of principal component analysis (PCA) exercised on growth and yield attributing characters of rice grain yield explicated the data variation to the tune of $90.15 \%$ of the total variability (Table 5). 
Three dominant principal components with Eigenvector less than 1 are extracted viz, PC1, PC 2 and PC3 accounting for $44.16 \%, 31.32 \%$ and $14.68 \%$ of cumulative variance in the available data set were extracted as an outcome of PCA (Table 5). Biplot (Fig. 1) generated in respect of rice elucidated that first principal component had large positive loadings number of tillers/hill, followed by effective tillers/hill, test weight and number of leaves/hill and these soil parameters exhibited high correlation between them since the variables of 0 or $180^{\circ}$ reveals a correlation of 1 or -1 , respectively (Kohler and Luniak, 2005). Growth and yield attributes (parameters) are superimposed on various $\mathrm{N}$ management practices $(\mathrm{N})$ and moisture regimes (treatments) on the biplots (Fig. 1) to figure out the best treatment combination affecting the growth and yield attributing characters and grain yield rice. The outcome of the superimposition showed that treatment N3M3 (50\% RDN through Urea $+25 \%$ RDN through FYM $+25 \%$ RDN through Azolla)+ $5 \mathrm{~cm}$ water depth at tillering, panicle initiation and flowering stages) was realized to be the best treatment combination followed by treatment combinations $50 \%$ RDN through Urea $+25 \%$ RDN through FYM $+25 \%$ through Azolla under no standing water and 50\% RDN through Urea $+25 \%$ RDN through FYM $+25 \%$ through Azolla under continuous flooding, respectively in influencing the growth and yield attributing characters and rice grain yield.

Stepwise multiple linear regressions (MLR) for predicting the best model for rice crop yield

For implementing MLR model on rice crop, the growth and yield attributing parameters were assigned as predictor variables and yield of rice crop were assigned as response variable to evaluate the main factors as the determinants of rice yield. Upon iteration, the following regression equation (Table 6) was generated after screening out the redundant predictor variables which were not involved directly in increasing rice crop yield. From the stepwise regression variances analysis (Table 7), it can be inferred that the yield attributing character i.e., number of tillers/hill was realized to be the best predictor variable impacting in augmenting the rice yield thereby contributing $66.46 \%$ followed by effective tillers/hill $(25.46 \%)$, test weight (4.21\%) and number of leaves/hill width ((3.44\%), respectively.

Table 6: Multiple stepwise linear regressions (MLR) equation for crops

$\begin{aligned} & \text { Grain yield }=16.18+1.05 \text { Number of tillers } / \text { hill }+6.85 \text { Test weight }+3.37 \text { Effective Tillers/hill }+0.04 \text { Number } \\
& \text { of leaves } / \text { hill }\end{aligned} \mid$\begin{tabular}{l|}
$R^{2}=99.57 \%$ \\
\hline$R^{2}$ adjusted $=99.13 \%$ \\
\hline$R^{2}$ predicted $=88.29 \%$ \\
\hline Durbin-Watson Statistic $=1.84$ \\
\hline
\end{tabular}

Table 7: Stepwise regression variances analysis

\begin{tabular}{|l|l|l|l|l|l|l|l|}
\hline Source & DF & Seq. SS & Contribution & Adj. SS & Adj. MS & F-Value & P-Value \\
\hline Regression & 4 & 6.22 & $99.57 \%$ & 6.22 & 1.55 & 229.11 & 0.00 \\
\hline Number of tillers/hill & 1 & 4.15 & $66.46 \%$ & 0.10 & 0.10 & 14.24 & 0.02 \\
\hline EffectiveTillers/hill & 1 & 1.59 & $25.46 \%$ & 1.79 & 1.79 & 264.08 & 0.00 \\
\hline Test weight of leaves/hill & 1 & 0.26 & $4.21 \%$ & 0.26 & 0.26 & 38.75 & 0.00 \\
\hline $\begin{array}{l}\text { Number } \\
\text { width }\end{array}$ & 1 & 0.21 & $3.44 \%$ & 0.03 & 0.03 & 3.72 & 0.13 \\
\hline Error & 4 & 0.03 & $0.43 \%$ & 0.03 & 0.01 & & \\
\hline Total & 8 & 6.24 & $100.00 \%$ & & & & \\
\hline
\end{tabular}

Where, Seq. SS = Sequential sums of squares, Adj. SS = Adjusted sum of squares, Adj. MS = Adjusted mean squares Stepwise selection of terms: $\alpha$ to enter $=0.15, \alpha$ to remove $=0.15$ 


\section{Conclusion}

Based on the above findings of pot experiment, it can be concluded that integrated approach of $\mathrm{N}$ management markedly improved growth and yield attributes and subsequently yield and water use efficiency of rice as compared to conventional method of $\mathrm{N}$ supplementation through fertilizer. Also, maintenance of $5 \mathrm{~cm}$ water depth at tillering, panicle initiation and flowering stage displayed a strong potential to replace the traditional practice of continuous flooding without affecting growth and yield of rice. So, it can be inferred that application of $50 \% \mathrm{RDN}$ through Urea $+25 \% \mathrm{RDN}$ through

\section{References}

Bisht, N., \& Chauhan, P. S. (2020). Excessive and Disproportionate Use of Chemicals Cause Soil Contamination and Nutritional Stress. In Soil Contamination. IntechOpen.

Boretti, A., \& Rosa, L. (2019). Reassessing the projections of the world water development report. NPJ Clean Water, 2(1), 1-6.

Jat, A. L., Srivastava, V. K., Chongtham, S. K., \& Singh, R. K. (2020). Integrated nitrogen management influences the yield performance of rice under different methods of cultivation. International Journal of Bio-resource and Stress Management, 11(5), 472-481.

Jat, A. L., Srivastava, V. K., Sen, A., Bohra, J. S., Maurya, B. R., \& Singh, R. K. (2016). Response of rice (Oryza sativa) hybrids to integrated nitrogen management under different methods of cultivation. Indian Journal of Agronomy, 61(3), 331-335.

Jeyajothi, R., \& Durairaj, S. N. (2015). Influence of integrated nutrient management practices on yield and yield attributes, and economics of transplanted Rice (Oryza ativa) in South Zone of Tamil Nadu. International Journal for Innovative Research in Science \& Technology, 1(11), 462-466.

Karkra, R., Kumar, P., Bansod, B. K., \& Krishna, C. R. (2016). Analysis of heavy metal ions in potable water using soft computing technique. Procedia Computer Science, 93, 988-994.

Kohler, U., \& Luniak, M. (2005). Data inspection using biplots. The Stata Journal, 5(2), 208-223.

Kumrawat, B., Verma, S. K., Singh, M. \&Argal, M. S. (2019). Effect of conjunctive use of organic and inorganic sources of nutrients on Soybean-Wheat productivity, economics and soil health of typic Ustochrepts of Central India. Indian Journal of Pure \& Applied Biosciences, 7(1), 563-573.
FYM $+25 \%$ through Azolla with maintenance of 5 $\mathrm{cm}$ depth at tillering, panicle initiation and flowering stages appears to be promising resource conserving practices for sustainable management of $\mathrm{N}$ and moisture in rice without comprising in terms of its growth and yield. However, to reach a specific conclusion and recommendation, the experiment should be tested under different agroclimatic situations and under open field conditions, with some due considerations on irrigation water quality and detailed study on the effect of nutrients on moisture stress tolerant capacity of rice.

Maji, A. T., \& Shaibu, A. A. (2012). Application of principal component analysis for rice germplasm characterization and evaluation. Journal of Plant Breeding and Crop Science, 4(6), 87-93.

Meshram, M. K., Dwivedi, B. S., Naik, K. R., Risikesh, T., \& Keram, K. S. (2018). Impact of organic and inorganic sources of nutrients on yield, nutrient uptake, soil fertility and economic performance of rice in a Typic haplustert. Journal of Soils and Crops, 28(1), 31-36.

Nataraja, T. H., Naika, R., Shankarappa, S. K., Reddy, K. V., Abdelmohsen, S. A., Al-Harbi, F. F., ... \& Abdelbacki, A M. (2021). Productivity of Paddies as Influenced by Varied Rates of Recommended Nutrients in Conjunction with Biofertilizers in Local Landraces. Agronomy, 11(6), 1165.

Oo, A. N., Banterng, P., Polthanee, A., \& Trelo-Ges, V. (2010). The effect of different fertilizers management strategies on growth and yield of upland black glutinous rice and soil property. Asian journal of plant sciences, 9(7), 414.

Pandey, N., Verma, A. K., \& Tripathi, R. S. (2010). Response of hybrid rice to scheduling of nitrogen and irrigation during dry season. ORYZA-An International Journal on Rice, 47(1), 34-37.

Ramakrishna, Y., Singh, S., \& Parihar, S. S. (2007). Influence of irrigation regime and nitrogen management on productivity, nitrogen uptake and water use by rice (Oryza sativa). Indian Journal of Agronomy, 52(2), 102-106.

Sharma, S., Padbhushan, R., \& Kumar, U. (2019). Integrated nutrient management in rice-wheat cropping system: an evidence on sustainability in the Indian subcontinent through meta-analysis. Agronomy, 9(2), 71.

Singh, S., \& Shivay, Y. S. (2003). Coating of prilled urea with ecofriendly neem (Azadirachta indica A. Juss.) formulations for efficient nitrogen use in hybrid rice. Acta agronomica hungarica, 51(1), 53-59. 
Thakur, A. K., Uphoff, N. T., \& Stoop, W. A. (2016). Scientific Underpinnings of the System of Rice Intensification (SRI): What is known so far?. Advances in agronomy, 135, 147-179.

Waraich, E. A., Ahmad, R., Ashraf, M. Y., Saifullah, \& Ahmad, M. (2011). Improving agricultural water use efficiency by nutrient management in crop plants. Acta Agriculturae Scandinavica, Section B-Soil \& Plant Science, 61(4), 291-304.

Xu, F., Song, T., Wang, K., Xu, W., Chen, G., Xu, M., \& Yuan, W. (2020). Frequent alternate wetting and drying irrigation mitigates the effect of low phosphorus on rice grain yield in a 4-year field trial by increasing soil phosphorus release and rice root growth. Food and Energy Security, 9(3), e206.

Yadav, L., \& Meena, R. N. (2014). Performance of aromatic rice (Oryza sativa) genotypes as influenced by integrated nitrogen management. Indian Journal of Agronomy, 59(2), 251-255.

Zhang, H., Xue, Y., Wang, Z., Yang, J., \& Zhang, J. (2009). An alternate wetting and moderate soil drying regime improves root and shoot growth in rice. Crop Science, 49(6), 2246-2260. 